\title{
EXISTENCE OF SOLUTIONS OF A SPECIAL CLASS OF FUZZY INTEGRAL EQUATIONS
}

\author{
K. BALACHANDRAN AND K. KANAGARAJAN
}

Received 30 July 2005; Revised 25 February 2006; Accepted 26 February 2006

We prove an existence theorem for a special class of fuzzy integral equations involving fuzzy set-valued mappings. The results are obtained by using the contraction mapping principle.

Copyright $\odot 2006$ K. Balachandran and K. Kanagarajan. This is an open access article distributed under the Creative Commons Attribution License, which permits unrestricted use, distribution, and reproduction in any medium, provided the original work is properly cited.

\section{Introduction}

Chandrasekhar [5] and Crum [6] considered the following integral equation:

$$
H(t)=1+H(t) \int_{0}^{1} \frac{t}{t+s} \psi(s) H(s) d s
$$

This equation arises in the study of radiation transfer in a semi-infinite atmosphere. The first rigorous proof of existence of solutions of (1.1) was given in [6]. By using operators on a Banach algebra and a fixed point theorem of Darbo for a set contraction map, Legget [8] proved an existence theorem for an equation of the form

$$
x=x_{0}+x K x,
$$

where $K$ is a compact operator on the Banach algebra. His abstract theorems are applied to the integral equation of the form

$$
x(t)=x_{0}(t)+x(t) \int_{\Omega} K(t, s) f(s, x(s)) d s, \quad t \in \Omega, \Omega \subset R^{n} .
$$


Cahlon and Eskin [4] considered the equation

$$
H(t)=1+H(t) \int_{0}^{1} \frac{t}{t+s} \psi(s) H(s) d s+\int_{0}^{1} P(t, s, H(t), H(s)) d s .
$$

This equation is a generalization of (1.3), where $P$ is the perturbation of Chandrasekhar $H$-equation.

The problem of existence of solutions of fuzzy integral equations has been studied by many authors [1, 2, 9-11, 14-16]. Kaleva [7] and Seikkala [13] have discussed the existence of solutions of fuzzy differential equations. Subrahmanyam and Sudarsanam [14] studied existence results for fuzzy Volterra integral equation of the form

$$
x(t)=\phi(t)+\int_{0}^{t} g(t, s, x(s)) d s
$$

where as Park et al. [11] proved the existence of solutions of fuzzy integral equation of the form

$$
\phi(u)=w_{0}+\int_{u_{0}}^{u} F(u, s, \phi(s)) d s \quad \phi\left(u_{0}\right)=w_{0} .
$$

Balachandran and Dauer [2] established the local existence of solutions and approximate solutions of the perturbed fuzzy integral equation. Balachandran and Prakash [3] studied the existence of solutions of nonlinear fuzzy Volterra integral equations of the form

$$
x(t)=\phi(t)+f\left(t, x(t), \int_{0}^{t} g(t, s, x(s)) d s\right) .
$$

In this paper we prove the existence of solutions of fuzzy integral equations of the form

$$
x(t)=\phi(t)+x(t) \int_{0}^{t} k(t, s) f(s, x(s)) d s+\int_{0}^{t} g(t, s, x(s)) d s,
$$

where $\phi:[0, T] \rightarrow E^{n}, k:[0, T] \times[0, T] \rightarrow R, f:[0, T] \times E^{n} \rightarrow E^{n}$, and $g:[0, T] \times[0, T] \times$ $E^{n} \rightarrow E^{n}$ are continuous functions. This equation is a generalization of Chandrasekhartype equation in fuzzy setting.

The outlay of the paper is as follows. In Section 2 we give some basic definitions for our study and in Section 3 we prove the main theorem on the existence of solutions of fuzzy integral equation (1.8). In Section 4 we state a theorem on the existence of solutions of a generalization of (1.8).

\section{Preliminaries}

Let $P_{k}\left(R^{n}\right)$ denote the family of all nonempty, compact, convex subsets of $R^{n}$. Addition and scalar multiplication in $P_{k}\left(R^{n}\right)$ are defined as usual. $\bar{U}$ denotes the closure of $U$, where $U$ is contained in $R^{n}$. Let $I=[0,1] \subseteq R$ be a compact interval and denote

$$
E^{n}=\left\{u: R^{n} \longrightarrow[0,1]: u \text { satisfies (i)-(iv) below }\right\}
$$


where

(i) $u$ is normal, that is, there exists an $x_{0} \in R^{n}$ such that $u\left(x_{0}\right)=1$,

(ii) $u$ is fuzzy convex,

(iii) $u$ is upper semicontinuous,

(iv) $[u]^{0}=\operatorname{cl}\left\{x \in R^{n}: u(x)>0\right\}$ is compact.

For $0<\alpha \leq 1$ denote $[u]^{\alpha}=\left\{x \in R^{n}: u(x) \geq \alpha\right\}$. Then from (i)-(iv) it follows that the $\alpha$-level set $[u]^{\alpha} \in P_{k}\left(R^{n}\right)$ for all $0 \leq \alpha \leq 1$.

If $g: R^{n} \times R^{n} \rightarrow R^{n}$ is a function, then using Zadeh's extension principle we can extend $g$ to $E^{n} \times E^{n} \rightarrow E^{n}$ by the equation

$$
\tilde{g}(u, v)(z)=\sup _{z=g(x, y)} \min \{u(x), v(y)\}
$$

It is well known that $[\tilde{g}(u, v)]^{\alpha}=g\left([u]^{\alpha},[v]^{\alpha}\right)$ for all $u, v \in E^{n}, 0 \leq \alpha \leq 1$, and continuous function $g$. In addition the above equation gives $[u+v]^{\alpha}=[u]^{\alpha}+[v]^{\alpha}$. The real numbers can be embedded in $E^{n}$ by the rule $c \rightarrow \hat{c}(t)$, where

$$
\widehat{c}(t)= \begin{cases}1 & \text { for } t=c \\ 0 & \text { elsewhere }\end{cases}
$$

We can also generalize the multiplication by a real number and for any real number $c$ we get $[c u]^{\alpha}=c[u]^{\alpha}$, where $0 \leq \alpha \leq 1$ and $u \in E^{n}$.

Let $D: E^{n} \times E^{n} \rightarrow R^{+} \cup\{0\}$ be defined by $D(u, v)=\sup _{0 \leq \alpha \leq 1} H\left([u]^{\alpha},[v]^{\alpha}\right)$, where $H$ is the Hausdorff metric defined in $P_{K}\left(R^{n}\right)$. Then $D$ is a metric on $E^{n}$. Further, $\left(E^{n}, D\right)$ is a complete metric space $[7,12]$. Also $D(u+w, v+w)=D(u, v)$ for every $u, v, w \in E^{n}$. Furthermore, $D(\lambda u, \lambda v)=|\lambda| D(u, v)$ for every $u, v \in E^{n}$ and $\lambda \in R$.

It can be proved straight away that $D(u+v, w+z) \leq D(u, w)+D(v, z)$ for $u, v, w$, and $z \in E^{n}$. (The proof is based on the fact $H\left(A_{1}+A_{2}, B_{1}+B_{2}\right) \leq H\left(A_{1}, B_{1}\right)+H\left(A_{2}, B_{2}\right)$, where $H$ is the Hausdorff metric on $P_{k}\left(R^{n}\right)$ induced by the norm in $R^{n}$.)

Definition 2.1 [1]. Let $I$ be $[0,1]$ and for each $t$ in $I$, let $F(t)$ be a nonempty subset of $R^{n}$. Let $\mathscr{F}$ be the set of all point-valued functions $f$ from $I$ to $R^{n}$ such that $f$ is integrable over $I$ and $f(t) \in F(t)$ for all $t$ in $I$. Then

$$
\int_{I} F(t) d t=\left\{\int_{I} f(t) d t: f \in \mathscr{F}\right\}
$$

Definition 2.2 [7]. A mapping $F: I \rightarrow E^{n}$ is strongly measurable if for all $\alpha \in[0,1]$ the set-valued map $F_{\alpha}: I \rightarrow P_{k}\left(R^{n}\right)$ defined by $F_{\alpha}(t)=[F(t)]^{\alpha}$ is Lebesgue measurable when $P_{k}\left(R^{n}\right)$ has the topology induced by the Hausdorff metric $H$.

Definition 2.3 [7]. A mapping $F: I \rightarrow E^{n}$ is said to be integrably bounded if there is an integrable function $h$ such that $\|x\| \leq h(t)$ for every $x \in F_{0}(t)$. 
$4 \quad$ Fuzzy integral equations

Definition $2.4[12]$. The integral of a fuzzy mapping $F:[0,1] \rightarrow E^{n}$ is defined levelwise by

$$
\begin{aligned}
{\left[\int_{[0,1]} F(t) d t\right]^{\alpha} } & =\int_{[0,1]} F_{\alpha}(t) d t \\
& =\left\{\int_{[0,1]} f(t) d t: f:[0,1] \longrightarrow R^{n} \text { is a measurable selection for } F_{\alpha}\right\}
\end{aligned}
$$

for all $\alpha \in[0,1]$.

It has been proved by Puri and Ralescu [12] that a strongly measurable and integrably bounded mapping $F: I \rightarrow E^{n}$ is integrable (i.e., $\int_{I} F(t) d t \in E^{n}$ ). The concept of a fuzzy integral generalizes the Aumann integral of a set-valued mapping. The following results are proved in [7].

Theorem 2.5. If $F: I \rightarrow E^{n}$ is continuous, then it is integrable.

Theorem 2.6. Let $F, G: I \rightarrow E^{n}$ be integrable and $\lambda \in R$. Then

(i) $\int_{I}(F(t)+G(t)) d t=\int_{I} F(t) d t+\int_{I} G(t) d t$,

(ii) $\int_{I} \lambda F(t) d t=\lambda \int_{I} F(t) d t$,

(iii) $D(F, G)$ is integrable,

(iv) $D\left(\int_{I} F(t) d t, \int_{I} G(t) d t\right) \leq \int_{I} D(F(t), G(t)) d t$.

\section{Existence theorem}

Theorem 3.1. Let $a$ and $b$ be positive numbers such that

$$
b=\max _{0 \leq t \leq a} D\left(\psi(t) \int_{0}^{t} k(t, s) f(s, \psi(s)) d s+\int_{0}^{t} g(t, s, \psi(s)) d s, \widehat{0}\right) .
$$

Suppose that

(i) $\phi:[0, a] \rightarrow E^{n}$ is continuous,

(ii) $f:[0, T] \times E^{n} \rightarrow E^{n}$ and $k:[0, T] \times[0, T] \rightarrow R$ are continuous and there exists $a$ constant $L>0$ such that

$$
D\left(x(t) \int_{0}^{t} k(t, s) f(s, x(s)) d s, y(t) \int_{0}^{t} k(t, s) f(s, y(s)) d s\right) \leq L D(x, y)
$$

for $x, y \in E^{n}$,

(iii) $g: U \rightarrow E^{n}$ is continuous, where $U=\left\{(t, s, x): 0 \leq s \leq t \leq a, x \in E^{n}\right.$ and $D(x, \phi(t)) \leq$ $b$ ) and satisfies Lipschitz condition with respect to $x$ on $U$, that is, there exists a constant $M>0$ such that

$$
D(g(t, s, x), g(t, s, y)) \leq M D(x, y) \quad \text { if }(t, s, x),(t, s, y) \in U
$$

If $c=(\alpha-L) / M$ for some fixed $\alpha \in(0,1)$, then there is a unique solution of $(1.7)$ on $[0, T]$, where $T=\min \{a, b, c\}$. 
Proof. Let $\mathscr{C}$ be the space of continuous functions from $[0, T]$ into $\left(E^{n}, D\right)$ with $H_{1}(\psi, \phi) \leq$ $b$, that is, $\mathscr{C}=\left\{\psi: \psi:[0, T] \rightarrow E^{n}\right.$ is continuous and $\left.H_{1}(\psi, \phi) \leq b\right\}$, where $H_{1}(\psi, \phi)=$ $\sup _{0 \leq t \leq T} D(\psi(t), \phi(t))$. Define an operator $A: \mathscr{C} \rightarrow \mathscr{C}$ by

$$
A \psi(t)=\phi(t)+\psi(t) \int_{0}^{t} k(t, s) f(s, \psi(s)) d s+\int_{0}^{t} g(t, s, \psi(s)) d s
$$

To prove $A: \mathscr{C} \rightarrow \mathscr{C}$, we have to prove that $A \psi$ is continuous and $H_{1}(A \psi, \phi) \leq b$ whenever $\psi \in \mathscr{C}$. Consider

$$
\begin{aligned}
& D(A \psi(t+h), A \psi(t)) \\
&=D\left(\phi(t+h)+\psi(t+h) \int_{0}^{t+h} k(t+h, s) f(s, \psi(s)) d s+\int_{0}^{t+h} g(t+h, s, \psi(s)) d s, \phi(t)\right. \\
&\left.\quad+\psi(t) \int_{0}^{t} k(t, s) f(s, \psi(s)) d s+\int_{0}^{t} g(t, s, \psi(s)) d s\right) \leq D(\phi(t+h), \phi(t)) \\
&+D\left(\psi(t+h) \int_{0}^{t+h} k(t+h, s) f(s, \psi(s)) d s, \psi(t) \int_{0}^{t} k(t, s) f(s, \psi(s)) d s\right) \\
&+\quad D\left(\int_{0}^{t+h} g(t+h, s, \psi(s)) d s, \int_{0}^{t} g(t, s, \psi(s)) d s\right) \\
& \leq+D\left(\psi(t+h) \int_{0}^{t+h} k(t+h, s) f(s, \psi(s)) d s, \psi(t) \int_{0}^{t} k(t, s) f(s, \psi(s)) d s\right) \\
&+\int_{0}^{t} D(g(t+h, s, \psi(s)) d s, g(t, s, \psi(s))) d s+\int_{t}^{t+h} D(g(t+h, s, \psi(s)) d s, \hat{0}) d s .
\end{aligned}
$$

Clearly the right-hand side of (3.5) is less than $\epsilon$ as $h \rightarrow 0$. So $A \psi$ is continuous. Consider

$$
\begin{aligned}
H_{1}(A \psi, \phi) & =\sup _{0 \leq t \leq T} D(A \psi(t), \phi(t)) \\
& =\sup _{0 \leq t \leq T} D\left(\phi(t)+\psi(t) \int_{0}^{t} k(t, s) f(s, \psi(s)) d s+\int_{0}^{t} g(t, s, \psi(s)) d s, \phi(t)\right) \\
& =\sup _{0 \leq t \leq T} D\left(\psi(t) \int_{0}^{t} k(t, s) f(s, \psi(s)) d s+\int_{0}^{t} g(t, s, \psi(s)) d s, \hat{0}\right) \\
& \leq b .
\end{aligned}
$$

So $A \psi \in \mathscr{C}$ and $A$ maps $\mathscr{C}$ into itself. We show that $\mathscr{C}$ is a closed subset of $C\left([0, T], E^{n}\right)$ a complete metric space with the metric $H_{1}$ (see [7]). 
Let $\left(\psi_{n}\right)$ be a sequence in $\mathscr{C}$ converging to $\psi$ in $C\left([0, T], E^{n}\right)$. Consider

$$
\begin{aligned}
H_{1}(\psi, \phi) & =\sup _{0 \leq t \leq T} D(\psi(t), \phi(t)) \\
& =\sup _{0 \leq t \leq T}\left\{D\left(\psi_{n}(t), \psi(t)\right)+D\left(\psi_{n}(t), \phi(t)\right)\right\} \\
& \leq H_{1}\left(\psi_{n}, \psi\right)+H_{1}\left(\psi_{n}, \phi\right) \\
& \leq \epsilon+b
\end{aligned}
$$

for sufficiently large $n$ and all positive $\epsilon$. So $\psi \in \mathscr{C}$. This implies that $\mathscr{C}$ is a closed subset of $C\left([0, T], E^{n}\right)$. Therefore $\mathscr{C}$ is a complete metric space. We prove that $A$ is a contraction mapping. For $\psi_{1}, \psi_{2} \in \mathscr{C}$,

$$
\begin{aligned}
& H_{1}\left(A \psi_{1}, A \psi_{2}\right) \\
& =\sup _{0 \leq t \leq T} D\left(A \psi_{1}(t), A \psi_{2}(t)\right) \\
& =\sup _{0 \leq t \leq T} D\left(\phi(t)+\psi_{1}(t) \int_{0}^{t} k(t, s) f\left(s, \psi_{1}(s)\right) d s+\int_{0}^{t} g\left(t, s, \psi_{1}(s)\right) d s, \phi(t)\right. \\
& \left.\quad+\psi_{2}(t) \int_{0}^{t} k(t, s) f\left(s, \psi_{2}(s)\right) d s+\int_{0}^{t} g\left(t, s, \psi_{2}(s)\right) d s\right) \\
& \left.\quad+\int_{0 \leq t \leq T}^{t} D\left(g\left(t, s, \psi_{1}(s)\right), g\left(t, s, \psi_{2}(s)\right)\right) d s\right\} \\
& \leq(L+M T) H_{1}\left(\psi_{1}, \psi_{2}\right) \\
& \leq(L+M c) H_{1}\left(\psi_{1}, \psi_{2}\right) \\
& \left.\leq \alpha(t, s) f\left(s, \psi_{1}(s)\right) d s, \psi_{2}(t) \int_{0}^{t} k(t, s) f\left(s, \psi_{2}(s)\right) d s\right) \\
& \leq H_{1}\left(\psi_{1}, \psi_{2}\right) \quad \text { where } \alpha \in(0,1) .
\end{aligned}
$$

So $A: \mathscr{C} \rightarrow \mathscr{C}$ is a contraction map. Since $\mathscr{C}$ is a complete metric space and $A$ is a contracting self-map on $\mathscr{C}$, it has a unique fixed point $x \in \mathscr{C}$. This fixed point is the required unique solution to (1.8).

\section{General equations}

As a generalization of (1.8) we consider the following fuzzy integral equation:

$$
x(t)=\phi(t)+h(t, x(t)) \int_{0}^{t} k(t, s) f(s, x(s)) d s+\int_{0}^{t} g(t, s, x(s)) d s,
$$

where $h:[0, T] \times E^{n} \rightarrow E^{n}$ is continuous and all other conditions are as before. Now we state without proof an existence theorem for (4.1). 
Theorem 4.1. Let $a^{*}$ and $b^{*}$ be positive numbers such that

$$
b^{*}=\max _{0 \leq t \leq a^{*}} D\left(h(t, \psi(t)) \int_{0}^{t} k(t, s) f(s, \psi(s)) d s+\int_{0}^{t} g(t, s, \psi(s)) d s, \hat{0}\right) .
$$

Suppose that

(i) $\phi:\left[0, a^{*}\right] \rightarrow E^{n}$ is continuous,

(ii) $f, h:[0, T] \times E^{n} \rightarrow E^{n}$ and $k:[0, T] \times[0, T] \rightarrow R$ are continuous and there exists a constant $L^{*}>0$ such that

$$
\begin{aligned}
& D\left(h(t, x(t)) \int_{0}^{t} k(t, s) f(s, x(s)) d s, h(t, y(t)) \int_{0}^{t} k(t, s) f(s, y(s)) d s\right) \\
& \quad \leq L^{*} D(x, y) \quad \text { for } x, y \in E^{n},
\end{aligned}
$$

(iii) $g: U \rightarrow E^{n}$ is continuous where $U=\left\{(t, s, x): 0 \leq s \leq t \leq a^{*}, x \in E^{n}\right.$ and $D(x$, $\left.\phi(t)) \leq b^{*}\right\}$ and satisfies Lipschitz condition with respect to $x$ on $U$, that is, there exists a constant $M^{*}>0$ such that

$$
D(g(t, s, x), g(t, s, y)) \leq M^{*} D(x, y) \quad \text { if }(t, s, x),(t, s, y) \in U
$$

If $c^{*}=\left(\alpha-L^{*}\right) / M$ for some fixed $\alpha \in(0,1)$, then there is a unique solution of (4.1) on $[0, T]$, where $T=\min \left\{a^{*}, b^{*}, c^{*}\right\}$.

\section{References}

[1] R. J. Aumann, Integrals of set-valued functions, Journal of Mathematical Analysis and Applications 12 (1965), no. 1, 1-12.

[2] K. Balachandran and J. P. Dauer, Existence of solutions of perturbed fuzzy integral equations in Banach spaces, Indian Journal of Pure and Applied Mathematics 28 (1997), no. 11, 1461-1468.

[3] K. Balachandran and P. Prakash, Existence of solutions of nonlinear fuzzy Volterra integral equations, Bulletin of the Calcutta Mathematical Society 94 (2002), no. 3, 147-152.

[4] B. Cahlon and M. Eskin, Existence theorems for an integral equation of the Chandrasekhar Hequation with perturbation, Journal of Mathematical Analysis and Applications 83 (1981), no. 1, 159-171.

[5] S. Chandrasekhar, The transfer of radiation in stellar atmospheres, Bulletin of the American Mathematical Society 53 (1947), 641-711.

[6] M. M. Crum, On an integral equation of Chandrasekhar, The Quarterly Journal of Mathematics. Oxford. Second Series 18 (1947), 244-252.

[7] O. Kaleva, Fuzzy differential equations, Fuzzy Sets and Systems 24 (1987), no. 3, 301-317.

[8] R. W. Leggett, On certain nonlinear integral equations, Journal of Mathematical Analysis and Applications 57 (1977), no. 2, 462-468.

[9] J. Mordeson and W. Newman, Fuzzy integral equations, Information Sciences 87 (1995), no. 4, 215-229.

[10] J. Y. Park and H. K. Han, Existence and uniqueness theorem for a solution of fuzzy Volterra integral equations, Fuzzy Sets and Systems 105 (1999), no. 3, 481-488.

[11] J. Y. Park, Y. C. Kwun, and J. U. Jeong, Existence of solutions of fuzzy integral equations in Banach spaces, Fuzzy Sets and Systems 72 (1995), no. 3, 373-378.

[12] M. L. Puri and D. A. Ralescu, Fuzzy random variables, Journal of Mathematical Analysis and Applications 114 (1986), no. 2, 409-422. 
[13] S. Seikkala, On the fuzzy initial value problem, Fuzzy Sets and Systems 24 (1987), no. 3, 319-330.

[14] P. V. Subrahmanyam and S. K. Sudarsanam, A note on fuzzy Volterra integral equations, Fuzzy Sets and Systems 81 (1996), no. 2, 237-240.

[15] _ An existence theorem for a fuzzy functional integral equation, Journal of Fuzzy Mathematics 5 (1997), no. 3, 723-732.

[16] C. Wu, S. Song, and H. Wang, On the basic solutions to the generalized fuzzy integral equation, Fuzzy Sets and Systems 95 (1998), no. 2, 255-260.

K. Balachandran: Department of Mathematics, Bharathiar University,

Coimbatore 641046, India

E-mail address: :balachandran_k@lycos.com

K. Kanagarajan: Department of Mathematics, Karpagam College of Engineering,

Coimbatore 641032, India

E-mail address: kanagarajank@rediffmail.com 


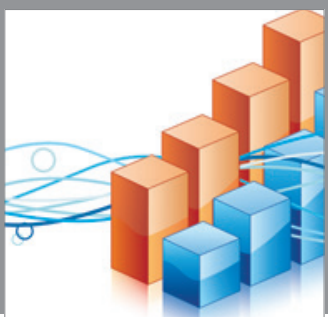

Advances in

Operations Research

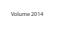

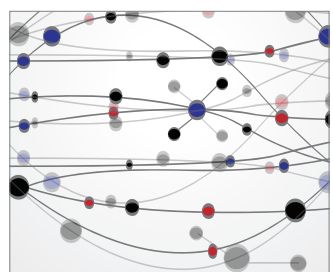

\section{The Scientific} World Journal
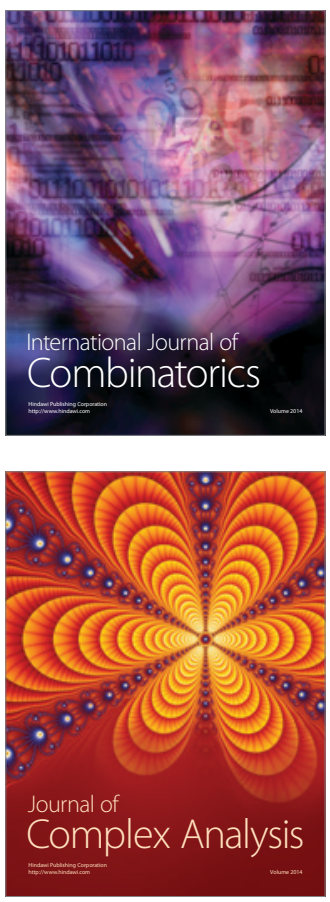

International Journal of

Mathematics and

Mathematical

Sciences
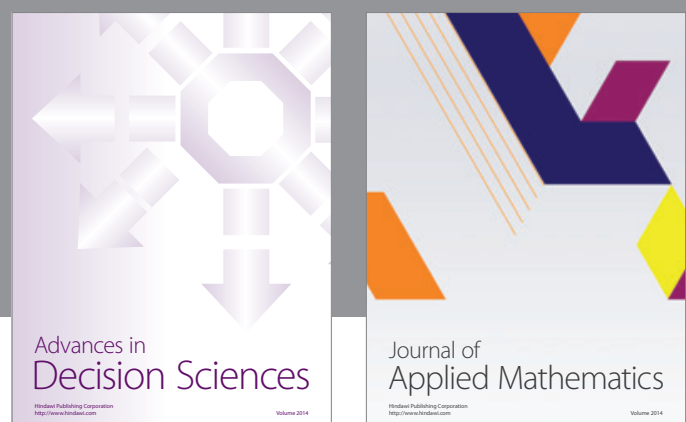

Journal of

Applied Mathematics
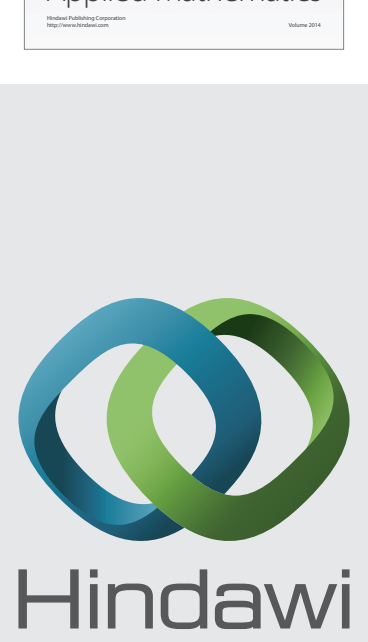

Submit your manuscripts at http://www.hindawi.com
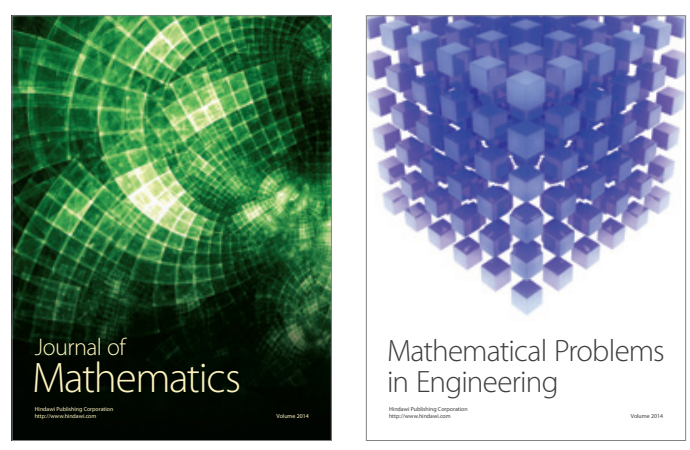

Mathematical Problems in Engineering
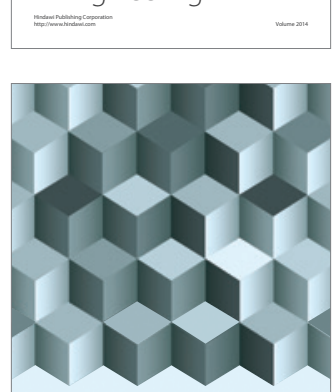

Journal of

Function Spaces
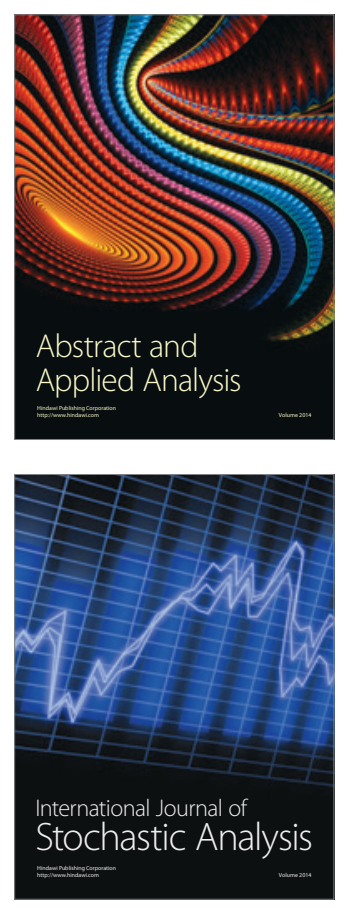

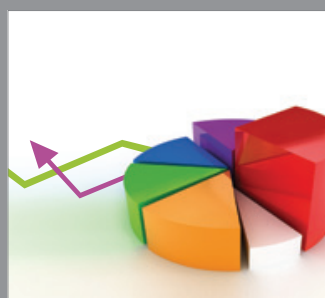

ournal of

Probability and Statistics

Promensencen
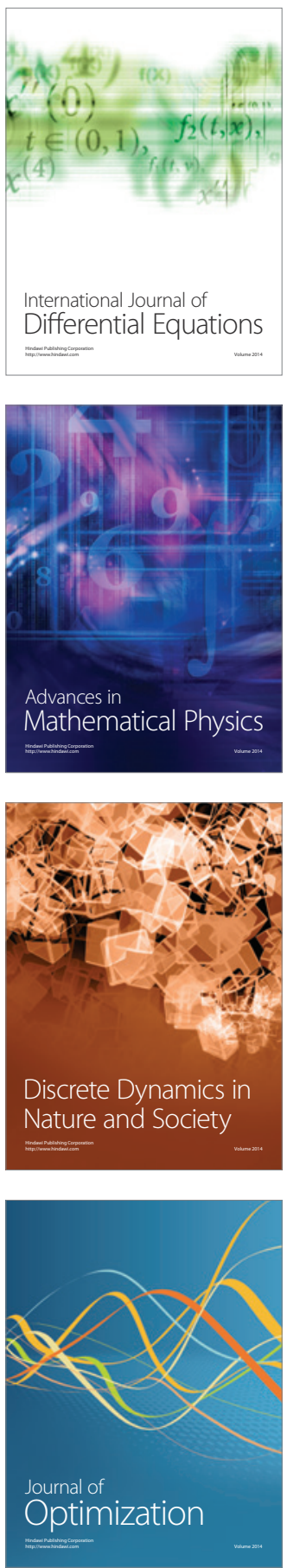